\title{
An overview on the role of autophagy in cancer therapy
}

\author{
Daniela Grácio ${ }^{1, a}$, Fernando Magro ${ }^{1, a}$, Raquel T. Lima ${ }^{2,3,4}$ and Valdemar Máximo ${ }^{2,3,4 *}$ \\ ${ }^{1}$ Departmento de Biomedicina, Faculdade de Medicina da Universidade do Porto, 4200-319 Porto, Portugal \\ ${ }^{2}$ Instituto de Investigação e Inovação em Saúde (i3S), Universidade do Porto, 4200-135 Porto, Portugal \\ ${ }^{3}$ Instituto de Patologia e Imunologia Molecular da Universidade do Porto (IPATIMUP), 4200-135 Porto, Portugal \\ ${ }^{4}$ Departmento de Patologia, Faculdade de Medicina da Universidade do Porto, 4200-319 Porto, Portugal \\ ${ }^{a}$ Contributed equally to this work, and should be considered joint first authors.
}

\begin{abstract}
Autophagy is a highly regulated catabolic process through which cells recycle their own constituents by delivering them into lysosomes. Several studies have demonstrated that autophagy plays a wide variety of physiological and pathophysiological roles in cells. In cancer, autophagy has been described to have paradoxical roles, acting both as tumor suppressor and as tumor promoter. In particular, it may exert different functions in response to cancer therapy, causing cancer resistance or increasing sensitivity to chemotherapeutic drugs and radiation. Therefore, autophagy could provide new means for the enhancement of antitumor drugs and radiation effectiveness.
\end{abstract}

\section{Introduction}

Autophagy (self-eating) is a highly conserved catabolic process with critical functions in the maintenance of cellular homeostasis under normal growth conditions and in the preservation of cell viability under stress [1]. Autophagy is an intracellular process in which cellular components, such as proteins and organelles, are delivered to the lysosome leading to the degradation and recycling of cytosolic compounds, thus providing cells with essential amino acids, nucleotides, and fatty acids, that enable production of elements required for energy and macromolecule biosynthesis [2,3]. There are three main types of autophagy, differing mainly in the mechanism by which the cytosolic material is presented to the lysosome [1]: i) macroautophagy, ii) microautophagy and iii) chaperone-mediated autophagy (CMA). In macroautophagy, double-membrane vesicles, called autophagosomes, sequester cytosolic material. Those vesicles merge with lysosomes (forming the autophagolysosome), their cargo is degraded, by lysosomal hydrolases, and the recycled macromolecular precursors are transported back into the cytoplasm, where they can be used as metabolic intermediates. In microautophagy, no intermediary vesicles are present, and the cytoplasmic material is directly engulfed by the lysosome [2]. In CMA, specific proteins, associated to heat shock protein (HSP) hsc70 and its co-chaperones, are translocated to the lysosome. Those proteins contain a specific amino acid motif (KFERQ, or biochemically related), which is recognized by the HSP, and once unfolded, they are translocated directly into the lysosome, via the lysosome-associated membrane protein 2A (LAMP2A) $[4,5]$

Several studies have already demonstrated that autophagy plays more roles than the initially expected, including: cellular adaptation to starvation, intracellular protein and organelle clearance, development, anti-aging, elimination of microorganisms, cell death and antigen presentation [6].

Deregulation of autophagy has been associated to several diseases, including neurodegenerative diseases, diabetes and cancer [7].
In this short review, we will mainly address the role of autophagy (and its different functional forms) in cancer, and its implication in cancer therapy. The majority of the studies published on autophagy, particularly those related to cancer therapy refer to "macroautophagy". In fact, the broad term 'autophagy' usually means "macroautophagy", unless otherwise specified, and therefore, in this review, we will also use this terminology [1]. Nevertheless, it is important to mention that recent studies have shown that CMA may be also important for tumor growth, progression and therapy and that pharmacological approaches that inhibit macroautophagy may also affect CMA $[8,9]$.

\section{Autophagy in cancer}

Cancer was one of the first diseases to be associated to autophagy [10-14]. Nevertheless, the exact molecular mechanisms and the role of autophagy in cancer cells is not yet clearly defined, being even paradoxical. While at early stages, autophagy usually acts as a tumor suppressor allowing cells to discard damaged cellular contents, decreasing ROS and DNA damage, in more advanced stages of tumor development, it may help cancer cells to survive under lowoxygen and low-nutrient conditions, acting as a tumor promoter $[3,15]$. Actually, the dependence of tumor cells on autophagy is highly variable. While some tumor models (like pancreatic cancer) display increased autophagy levels in basal situations (including in plenty nutrient conditions), with autophagy having a role in the maintenance of tumor growth [16], results from other studies, comparing the levels of autophagy in tumor cells with their corresponding non-tumor cells, show disparate data between different tumor models (for a thorough

Correspondence to: Valdemar Máximo, Cancer Signaling and Metabolism research group, Instituto de Patologia e Imunologia Molecular da Universidade do Porto (IPATIMUP), 4200-135 Porto, Portugal, Tel: 225570 700; Fax: 225570 799; E-mail: vmaximo@ipatimup.pt

Received: January 24, 2017; Accepted: February 16, 2017; Published: February 18,2017 
review please see [17]).

Importantly, autophagy plays also a role in cancer response to therapy since cancer therapies mostly inflict stress and damage to cells to induce cell death [18]. The outcomes of therapy-induced autophagy in cancer cells may represent also a "double-edge sword" and depends on the particular type of cancer, on the stage of disease progression or even on the type and duration of autophagy [18-21]. Indeed, several studies showed that increased autophagy leads to resistance to both chemo- and radiotherapy, while several others show that many anticancer drugs induce autophagy-related cell death in cancer cells $[22,23]$.

The fact that many of the currently used clinically approved anticancer strategies have been described as inducing autophagy, makes the understanding of the functional role of autophagy within a specific cancer context much more relevant, as it could provide new means for the enhancement of antitumor drugs and radiation effectiveness.

\section{Functional forms of autophagy and their implications for cancer therapy}

Although, traditionally, autophagy has been seen as a pro-survival (cytoprotective) mechanism, different studies have shown that it may result in other outcomes. Currently, at least four distinct functional forms of autophagy have been described [24,25]: i) Cytoprotective, when cells die or arrest if autophagy is inhibited; ii) Cytotoxic, when autophagy induction results in cell death and its blockage results in cell survival; iii) Cytostatic, when autophagy induction results in cell growth arrest and iv) Nonprotective, if autophagy does not affect cell growth once blocked. These forms are distinguished on only based on their functional characteristics, having similar morphologic, biochemical or molecular profiles [24].

\section{Autophagy modulation as a therapeutic strategy to im- prove anticancer strategies}

As already referred, the different functional forms of autophagy affect the cellular response to anticancer therapies. The knowledge whether autophagy is cytoprotetive or is cytotoxic/cytostatic, will help defining strategies for its modulation (through its decrease or increase, respectively) to interfere with the cellular sensitivity to therapy.

Targeting cytoprotective autophagy has been at the basis for multiple clinical trials. Indeed, if increased autophagy confers tumor resistance to death-inducing agents, its inhibition will allow an enhanced response to treatment [26]. There are several autophagy inhibitors already identified and that have been classified as: earlystage inhibitors, if blocking autophagosome formation [such as 3-Methyladenine (3-MA), wortmannin, and LY294002] orlate-stage inhibitors, acting at the level of the autophagosome-lysosome fusion and degradation steps [such as chloroquine (CQ), hydroxychloroquine (HCQ), bafilomycin A1, and monensin]. Studies using, not only these pharmacological autophagy inhibitors, but also genetic silencing or knockdown of autophagy-associated genes, resulted in increased tumor cell sensitivity to the autophagy-inducing stimulus, usually via the promotion of apoptosis $[24,26]$.

Several clinical trials have been evaluating the use of autophagy inhibitors (particularly HCQ) in combination to chemo- and radiotherapy to improve its efficacy $[27,28]$. A study carried out in melanoma patients using HCQ in combination with the mTOR inhibitor (temsirolimus) showed an improvement of the median progression- free survival to 3.5 months and increased the rate of stable disease in patients $[27,29]$. Also, its combination with a proteasome inhibitor (bortezomib) in relapsed/refractory myeloma patients resulted in a higher rate of partial response and stable disease [30]). More recently, the use of HCQ in combination with gemcitabine in pancreatic ductal adenocarcinoma patients caused significant decreases in the disease biomarker, CA 19-9, with the mean overall survival being extended to nearly 3 years $[28,31]$. Although clinical trials with these compounds indicate that autophagy inhibition in patients is possible, there is still room for improvement, since CQ/HCQ have also shown significant variability of autophagy inhibition levels among patients. Moreover, these type of compounds, although being already FDA approved, have to be administered in higher concentrations to inhibit autophagy and are retained for long periods of time in patients (some studies showing patients retaining HCQ) in their system up to 5 years [28,32].

On the other hand, autophagy induction may help improve the effect of anticancer therapies when autophagy is cytotoxic, by inducing cell death by itself or by the activation of other cell death mechanism, namely apoptosis $[33,34]$. Several drugs/natural extracts, some of which already used in the clinic, have been described to induce autophagymediated cell death in different cancer cells [23]. For example, the combination of Vitamin D with radiation promoted cytotoxic autophagy in breast tumor cells [35,36]. Resveratrol and curcumin caused cell death in several human tumor cell lines through apoptosis and autophagy $[37,38]$. Naphthazarin, a naphthoquinone compound acting as microtubule depolymerizing agent was shown to induce cell death in lung cancer cells through apoptosis and autophagy [39]. In addition, the small molecule STF-62247 induced autophagic cell death in Von Hippel Lindau (VHL)-deficient renal cell carcinoma cells [40] and TXA1, a thioxantonic small molecule, decreased the viability of melanoma and breast cancer cells through the induction of autophagy [41].

\section{Autophagy in immunotherapy}

The role of immune response has been gaining particular interest for cancer therapy. Recently, autophagy has also been described as playing an important role in the regulation of immune recognition and response [42]. It has been demonstrated that autophagy increases tumor cells immunogenicity, being involved in tumor antigen processing and in the subsequent activation of the effector $\mathrm{T}$ cells. Thus, strategies aiming at autophagy induction could serve as adjuvant to stimulate the antitumor immune response. For example, the use of tumor autophagosome-derived vaccines have been found to induce cytotoxic immune cells and, consequently, antitumor activity in mice bearing lung carcinoma and melanoma cell lines [43].

Recent studies show that, since increased levels of autophagy in cancer cell suppresses the antitumor immune response, autophagy inhibition improves antitumor immune responsein immunotherapeutic strategies, such as adoptive transfer of T cells, vaccines, administration of antibodies or recombinant cytokines [44] Based on published findings, autophagy inhibition may increase the cytotoxicity of effector $\mathrm{T}$ and NK cells once they have been activated to lyse the tumor cells. The combination of high doses of IL-2 with chloroquine increased long term survival, decreased vascular leakage associated toxicity, and enhanced immune cell proliferation and infiltration in the liver and spleen [45]. Autophagy plays also a fundamental role in increasing the immunogenicity of the tumor cell, participates in the antigen processing and in the subsequent activation of the effector $\mathrm{T}$ cells, and its induction could be exploited as adjuvant strategy to stimulate the 
antitumor immune response $[43,46]$

The understanding under which circumstances inducers or inhibitors of autophagy affect the therapeutic efficacy of anticancer treatments will be important to improve the rational use of such modulators, since the data available do not yet allow us to realize this [46].

\section{Summary}

Autophagy plays an important role as a stress response mechanism to chemotherapeutic drugs and radiation in cancer cells. There are at least four functional forms of autophagy that may occur in response to chemotherapy or radiation: cytoprotective, nonprotective, cytotoxic and cytotastic. Currently, is not possible to predict which form will be induced by a particular therapy, since these forms of autophagy have no clear-cut morphologic, biochemical, or molecular distinctions. In some circumstances, autophagy protects tumor cells from cancer therapy while, in others it is associated with cancer cell killing. Modulation of autophagy may represent an important therapeutic opportunity to enhance the efficacy of anticancer therapies. The future challenge for autophagy research in cancer therapy is to find ways to identify which functional form of autophagy is activated, in specific tumor models, and which tumors may be most effectively treated by autophagy modulation. A better understanding of the role of autophagy in different tumor models will provide new therapeutic tools for more effective cancer therapeutic strategies.

\section{References}

1. Mizushima N (2007) Autophagy: process and function. Genes Dev 21: 2861-2873. [Crossref]

2. Thorburn A, Thamm DH, Gustafson DL (2014) Autophagy and cancer therapy. Mol Pharmacol 85: 830-838.[Crossref]

3. Cuervo AM (2004) Autophagy: in sickness and in health. Trends Cell Biol 14: 70-77. [Crossref]

4. Wang DW, Peng ZJ, Ren GF, Wang GX (2015) The different roles of selective autophagic protein degradation in mammalian cells. Oncotarget6: 37098-37116. [Crossref]

5. Parzych KR, Klionsky DJ (2014) An overview of autophagy: morphology, mechanism, and regulation. Antioxid Redox Signal 20: 460-473.[Crossref]

6. Mizushima N (2005) The pleiotropic role of autophagy: from protein metabolism to bactericide. Cell Death Differ 12 Suppl 2: 1535-1541.[Crossref]

7. Lorin S, Hamaï A, Mehrpour M, Codogno P (2013) Autophagy regulation and its role in cancer. Semin Cancer Biol 23: 361-379.[Crossref]

8. Kon M, Kiffin R, Koga H, Chapochnick J, Macian F, et al. (2011) Chaperone-mediated autophagy is required for tumor growth. Sci Transl Med 3: 109ra117.[Crossref]

9. Thorburn A, Debnath J (2011) Targeting chaperone-mediated autophagy in cancer. Sci Transl Med 3: 109ps45.[Crossref]

10. OmodeiZorini A (1965) Considerations on primary carcinomatous caverns of the lung. Possibility of the intervention of a phenomenon of "autophagy of the neoplastic cells". LottaTuberc35: 946-968.[Crossref]

11. Gullino PM, Lanzerotti RH (1972) Mammary tumor regression. II. Autophagy of neoplastic cells. J Natl Cancer Inst 49: 1349-1356.[Crossref]

12. Qu X, Yu J, Bhagat G, Furuya N, Hibshoosh H, et al. (2003) Promotion of tumorigenesis by heterozygous disruption of the beclin 1 autophagy gene. J Clin Invest 112: 18091820.[Crossref]

13. Yue Z, Jin S, Yang C, Levine AJ, Heintz N (2003) Beclin 1, an autophagy gene essential for early embryonic development, is a haploinsufficienttumor suppressor. Proc Natl Acad Sci U S A 100: 15077-15082.[Crossref]

14. Edinger AL, Thompson CB (2003) Defective autophagy leads to cancer. Cancer Cell 4: 422-424.[Crossref]

15. Lu Z, Luo RZ, Lu Y, Zhang X, Yu Q, et al. (2008) The tumor suppressor gene ARHI regulates autophagy and tumor dormancy in human ovarian cancer cells. $J$ Clin Invest 118: 3917-3929.[Crossref]

16. Yang S, Wang X, Contino G, Liesa M, Sahin E, et al. (2011) Pancreatic cancers require autophagy for tumor growth. Genes Dev 25: 717-729.[Crossref]

17. Ogier-Denis E, Codogno P (2003) Autophagy: a barrier or an adaptive response to cancer. Biochim Biophys Acta 1603: 113-128.[Crossref]

18. White E, DiPaola RS (2009) The double-edged sword of autophagy modulation in cancer. Clin Cancer Res 15: 5308-5316.[Crossref]

19. Towers CG, Thorburn A2 (2016) Therapeutic Targeting of Autophagy. EBio Medicine. [Crossref]

20. Levine B (2007) Cell biology: autophagy and cancer. Nature 446: 745-747.[Crossref]

21. Levine B, Kroemer G (2008) Autophagy in the pathogenesis of disease. Cell 132: 27 42. [Crossref]

22. Scarlatti F, Bauvy C, Ventruti A, Sala G, Cluzeaud F, et al. (2004) Ceramide-mediated macroautophagy involves inhibition of protein kinase B and up-regulation of beclin 1 . J Biol Chem 279: 18384-18391.[Crossref]

23. Eberhart K, Oral O, Gozuacik D (2014) Induction of Autophagic Cell Death by Anticancer Agents A2 - Hayat MA, in Autophagy: Cancer, Other Pathologies, Inflammation, Immunity, Infection, and Aging, Amsterdam P: 179-202.

24. Gewirtz DA (2014) The four faces of autophagy: implications for cancer therapy Cancer Res 74: 647-651.[Crossref]

25. Sharma K, Le N, Alotaibi M, Gewirtz DA (2014) Cytotoxic autophagy in cancer therapy. Int J Mol Sci 15: 10034-10051.[Crossref]

26. Carew JS1, Kelly KR, Nawrocki ST (2012) Autophagy as a target for cancer therapy: new developments. Cancer Manag Res 4: 357-365.[Crossref]

27. Zhi X, Zhong Q (2015) Autophagy in cancer. F1000Prime Rep 7: 18.[Crossref]

28. Solitro AR, MacKeigan JP (2016) Leaving the lysosome behind: novel developments in autophagy inhibition. Future Med Chem 8: 73-86.[Crossref]

29. Rangwala R, Chang YC, Hu J, Algazy KM, Evans TL, et al. (2014) Combined MTOR and autophagy inhibition: phase I trial of hydroxychloroquine and temsirolimus in patients with advanced solid tumors and melanoma. Autophagy 10: 1391-1402. [Crossref]

30. Vogl DT, Stadtmauer EA, Tan KS, Heitjan DF, Davis LE, et al. (2014) Combined autophagy and proteasome inhibition: a phase 1 trial of hydroxychloroquine and bortezomib in patients with relapsed/refractory myeloma. Autophagy 10: 1380-1390. [Crossref]

31. Boone BA, Bahary N, Zureikat A1, Moser AJ, Normolle DP, et al. (2015) Safety and Biologic Response of Pre-operative Autophagy Inhibition in Combination with Gemcitabine in Patients with Pancreatic Adenocarcinoma. Ann Surg Oncol 22: 4402 4410.[Crossref]

32. Browning DJ1 (2002) Hydroxychloroquine and chloroquine retinopathy: screening for drug toxicity. AmJ Ophthalmol 133: 649-656.[Crossref]

33. Sui X, Chen R, Wang Z, Huang Z, Kong N, et al. (2013) Autophagy and chemotherapy resistance: a promising therapeutic target for cancer treatment. Cell Death Dis 4: e838. [Crossref]

34. Zarzynska JM (2014) The importance of autophagy regulation in breast cancer development and treatment. Biomed Res Int 2014: 710345.[Crossref]

35. Wilson EN, Bristol ML, Di X, Maltese WA, Koterba K, et al. (2011) A switch between cytoprotective and cytotoxic autophagy in the radiosensitization of breast tumor cells by chloroquine and vitamin D. Horm Cancer 2: 272-285.[Crossref]

36. Bristol ML, Di X, Beckman MJ, Wilson EN, Henderson SC, et al. (2012) Dua functions of autophagy in the response of breast tumor cells to radiation: cytoprotective autophagy with radiation alone and cytotoxic autophagy in radiosensitization by vitamin D 3. Autophagy 8: 739-753.[Crossref]

37. Selvaraj S, Sun Y, Sukumaran P, Singh BB (2016) Resveratrol activates autophagic cell death in prostate cancer cells via downregulation of STIM1 and the mTOR pathway. MolCarcinog 55: 818-831.[Crossref]

38. Hasima N, Ozpolat B2 (2014) Regulation of autophagy by polyphenolic compounds as a potential therapeutic strategy for cancer. Cell Death Dis 5: e1509.[Crossref]

39. Acharya BR, Bhattacharyya S, Choudhury D, Chakrabarti G (2011) The microtubule depolymerizing agent naphthazarin induces both apoptosis and autophagy in A549 lung 


\section{cancer cells. Apoptosis 16: 924-939.[Crossref]}

40. Turcotte S, Chan DA, Sutphin PD, Hay MP, Denny WA, et al. (2008) A molecule targeting VHL-deficient renal cell carcinoma that induces autophagy. Cancer Cell 14 90-102.[Crossref]

41. Lima RT1,2,3, Sousa D4,5, et al. (2016) Modulation of Autophagy by a Thioxanthone Decreases the Viability of Melanoma Cells. Molecules21.[Crossref]

42. Pan H1,2, Chen L1,2, Xu Y1, et al. (2016) Autophagy-associated immune responses and cancer immunotherapy. Oncotarget 7: 21235-21246.[Crossref]

43. Li Y1, Wang LX, Pang P, Cui Z, Aung S, et al. (2011) Tumor-derived autophagosome vaccine: mechanism of cross-presentation and therapeutic efficacy. Clin Cancer Res 17: 7047-7057.[Crossref]

44. Amaravadi RK1, Lippincott-Schwartz J, Yin XM, Weiss WA, Takebe N, et al. (2011) Principles and current strategies for targeting autophagy for cancer treatment. Clin Cancer Res 17: 654-666.[Crossref]

45. Liang X (2012) Inhibiting systemic autophagy during interleukin 2 immunotherapy promotes long-term tumor regression. Cancer Res 72: 2791-2801.

46. Viry E, Paggetti J, Baginska J, Mgrditchian T, Berchem G, et al. (2014) Autophagy: an adaptive metabolic response to stress shaping the antitumor immunity. Biochem Pharmacol 92: 31-42.[Crossref]

Copyright: $@ 2017$ Grácio D. This is an open-access article distributed under the terms of the Creative Commons Attribution License, which permits unrestricted use, distribution, and reproduction in any medium, provided the original author and source are credited. 